\title{
Avaliação ecodopplercardiográfica da função diastólica em cães da raça Boxer
}

\section{RESUMO}

Foram avaliados os principais índices diastólicos, inclusive a velocidade de propagação mitral (Vp), em 36 cães sadios da raça Boxer, 18 de cada sexo, de idade entre um ano e meio e seis anos. A relação raiz aórtica e átrio esquerdo medida no modo $\mathrm{B}$ foi de $1,17 \pm 0,11$. Os picos de velocidades das ondas de enchimento atrial esquerdo foram: sistólica $31,41 \pm 6,87 \mathrm{~cm} / \mathrm{s}$, diastólica (D) $73,85 \pm 17,04 \mathrm{~cm} / \mathrm{s}$ e de reversão atrial $28,90 \pm 8,33 \mathrm{~cm} / \mathrm{s}$. A relação das ondas de enchimento ventricular inicial (E) e atrial (A) foi

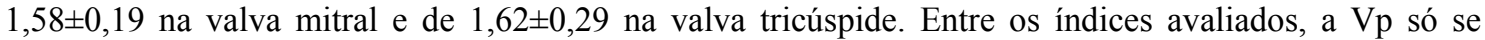
correlacionou fracamente $(\mathrm{r}=0,39) \mathrm{com}$ as ondas $\mathrm{E}$ e A mitrais e com a onda $\mathrm{D}$, mostrando-se um índice, relativamente, independente. A Vp não apresentou diferença significativa entre os sexos nem entre examinadores. A Vp diminuiu com o aumento da idade e exibiu valor médio, neste estudo, de $99,73 \pm 16,06 \mathrm{~cm} / \mathrm{s}$.

Palavras-chave: cão, Boxer, função diastólica, modo $\mathrm{M}$ em cores, fluxo das veias pulmonares, Vp

\begin{abstract}
Major diastolics indexes, including the mitral propagation velocity ( $\mathrm{Vp})$, were evaluated in 36 healthy Boxer dogs, 18 males and 18 females, aging from 1.5 to 6-years-old. The aortic root and left atrium ratio, measured in B mode, was 1.17 \pm 0.11 . The peaks of velocity of waves of the left atrium filling were: systolic $31.41 \pm 6.87 \mathrm{~cm} / \mathrm{s}$, diastolic $73.85 \pm 17.04 \mathrm{~cm} / \mathrm{s}$ and atrial reversal $28.90 \pm 8.33 \mathrm{~cm} / \mathrm{s}$. The ratios of the waves of initial ventricular filling were $1.58 \pm 0.19$ to mitral valve and $1.62 \pm 0.29$ to tricuspid valve. From the evaluated indexes, the Vp was only weakly correlated $(r=0.39)$ with $E$ and $A$ waves of mitral valve and with D, showing itself, relativity, as an independence index. Regarding to the Vp, no significant differences were seen between the gender of the animals and among the observers. The Vp decreased as the age increased, showing mean values of $99.73 \pm 16.06 \mathrm{~cm} / \mathrm{s}$.
\end{abstract}

Keywords: dog, Boxer, diastolic function, color M mode, pulmonary veins flow, Vp

\section{INTRODUÇÃO}

Até a década dos anos 90, o principal enfoque do exame ecocardiográfico era a função sistólica. Devido à necessidade de avaliação mais profunda, tem-se percebido que a disfunção diastólica geralmente precede a sistólica, influenciando significantemente na sintomatologia clínica e no prognóstico das cardiopatias.

Recebido em 16 de março de 2007

Aceito em 25 de setembro de 2007

*Autor para correspondência (corresponding autor)

E-mail: baracat@vet.ufmg.br 
$\mathrm{Na}$ doença cardíaca, há aumento da pré-carga e da freqüência cardíaca, levando à pseudonormalização dos índices diastólicos. Os recentes estudos ecodopplercardiográficos concentram-se em desmascarar a pseudonormalização e correlacionar as doenças cardíacas com padrões diastólicos ao ECO.

Estudos epidemiológicos, eletrocardiográficos e da função sistólica ao ECO realizados com 189 cães, com cardiomiopatia dilatada, não encontraram correlação entre raça, sexo, presença de arritmias, fração de ejeção (FE), espessura do septo interventricular, tamanho atrial e espessuras da parede livre e da parede septal do ventrículo esquerdo com a sobrevida. Os principais fatores influenciadores no prognóstico foram o início dos sinais clínicos e a idade de início da doença (Tidholm et al., 1997). Provavelmente, esses pesquisadores teriam encontrado relação da sobrevida com a função diastólica, caso a tivessem estudado.

Metade dos pacientes humanos com insuficiência cardíaca congestiva apresenta função sistólica preservada. O doppler tecidual e a medida da velocidade de propagação mitral ao modo $\mathrm{M}$ em cores (Vp) não sofrem alterações significativas com mudanças da pré-carga, sendo os métodos ecocardiográficos de escolha para a avaliação diastólica (Schober et al., 2003; Pedone et al., 2004). Não foi encontrado estudo de avaliação da Vp em cães.

O fluxo das veias pulmonares e o fluxo das veias cavas são usados na avaliação da função diastólica atrial, porém de obtenção difícil e maior variabilidade que os das valvas atrioventriculares. $\mathrm{O}$ estudo dos fluxos diastólicos atriais com doppler pulsado mostra três ondas: uma sistólica, uma diastólica e uma de reversão atrial (Wilkenshoff et al., 2001).

A diástole ventricular compreende o tempo entre o fechamento da valva aórtica e o da mitral. $\mathrm{O}$ doppler pulsado divide-se em dois momentos: 1tempo entre o fechamento da valva aórtica e abertura da valva atrioventricular, chamado de tempo de relaxamento isovolumétrico (TRIV) e 2 - tempo entre a abertura e o fechamento da valva atrioventricular, com duas ondas de enchimento diastólico: a onda $\mathrm{E}$ (enchimento inicial) e a A (enchimento tardio devido à contração atrial) (Nagueh et al., 1996).
O TRIV, os picos de velocidade das ondas E e A, o tempo de desaceleração da onda E (TDE) e a relação E/A são usados na avaliação transmitral ao doppler pulsado (Nagueh et al., 1996).

$\mathrm{O}$ modo $\mathrm{M}$ em cores (MC) dá a informação de três dimensões em duas, por meio da codificação de cores diferentes para velocidades diferentes; a passagem do sangue pela valva mitral é uma passagem de fluido por orifício (Mey et al., 2001).Um fluido, ao atravessar região de menor diâmetro, forma vórtices como o do anel de fumaça. Os vórtices apresentam um núcleo, um ânulo e velocidade angular (Vp). Os fluidos presos dentro do vórtice apresentam velocidade de rotação V (Fig. 1) (Mey et al., 2001).

Usando-se modelo hidráulico, demonstrou-se que a velocidade de deslocamento do vórtice é muito semelhante à $\mathrm{Vp}$ de enchimento ventricular e diminui no deslocamento ao ápice cardíaco. A Vp é mensurada através da rampa visibilizada na tela do aparelho de ecocardiografia (Mey et al., 2001).

Alterações na pré-carga e na freqüência cardíaca não mudam a Vp. Das medidas diastólicas ao ECO e medidas invasivas, a tau é a que mais se relaciona com a $\mathrm{Vp}(\mathrm{r}=0,75$ à 0,86$)$ (Garcia et al., 2000; Mey et al., 2001).

O método mais relacionado com o vórtice ventricular é o de Takatsuji et al. (1996), porém é influenciado pela freqüência cardíaca. $\mathrm{O}$ método utilizado por Brun et al. (1992) apresenta grande variação intra e interobservador, sendo a maneira proposta por Garcia et al. (2000) a mais usada nos recentes estudos da medicina humana (Mey et al., 2001; Palecek et al., 2004). A Vp pode ser medida na primeira mudança de cor do fluxo mitral ao MC (Garcia et al., 2000; Mey et al., 2001).

Os cães da raça Boxer são muito acometidos por doenças cardíacas, e as doenças mais observadas na raça são: cardiomiopatia dilatada, estenose aórtica e estenose pulmonar (Tidholm et al., 1997).

Este trabalho teve o objetivo de avaliar os padrões ecodopplercardiográficos da função diastólica em cães clinicamente sadios da raça Boxer, com ênfase na padronização da velocidade de propagação mitral $(\mathrm{Vp})$. 

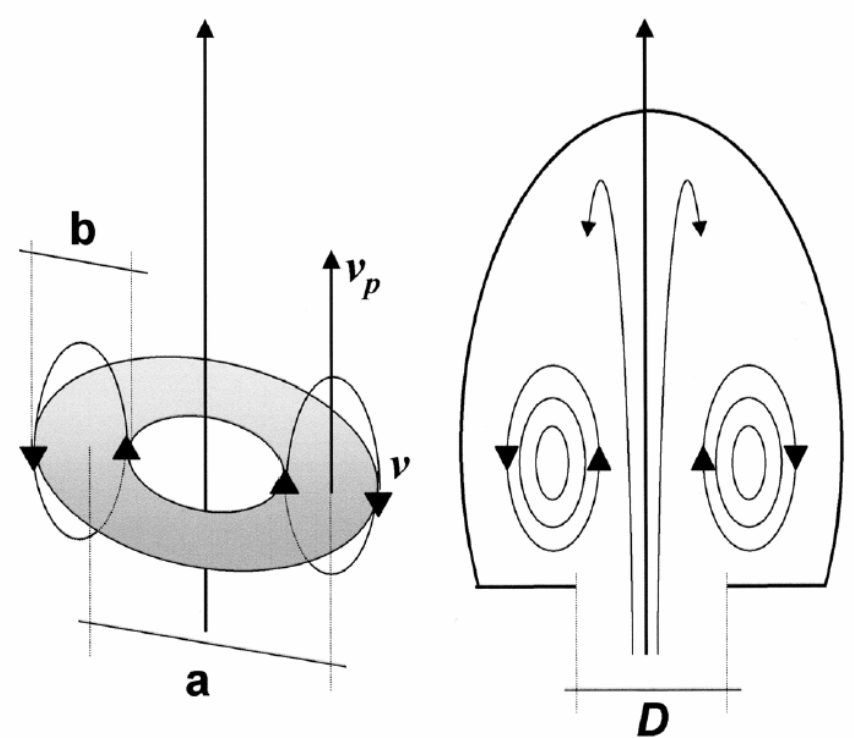

Figura 1. Princípio da formação do vórtice no enchimento ventricular. O vórtice é semelhante ao anel de fumaça (lado esquerdo): - (a) diâmetro do núcleo do anel; - (b) largura do vórtice; - (D) anel valvar atrioventricular. $\mathrm{O}$ vórtice propaga-se na direção do ápice cardíaco na velocidade $\mathrm{Vp}$, as partículas dentro do vórtice exibem velocidade V (Adaptado de Mey et al., 2001).

\section{MATERIAL E MÉTODOS}

Foram utilizados 18 cães e 18 cadelas, clinicamente normais, da raça Boxer, com idades entre um ano e meio e seis anos e média de peso de $27,18 \pm 4,28 \mathrm{~kg}$.

A avaliação da variação entre examinadores foi composta por 11 cães, sendo três machos e oito cadelas, com peso $25,12 \pm 4,66 \mathrm{~kg}$.

Todos os animais foram submetidos a exame físico, eletrocardiográfico, pressórico e ecocardiográfico rotineiro. Os resultados foram anotados em fichas individuais. Após exame clínico geral e específico, foram mensuradas as pressões sistólica, diastólica e média na região tibialdistal esquerda, por método oscilométrico com aparelho Dixtal DX 2010. Foi realizado o exame eletrocardiográfico (aparelho Ecafix 6), nas derivações I, II, III, aVR, aVL e aVF (velocidade de $50 \mathrm{~mm} / \mathrm{s}, \quad 1 \mathrm{~cm}=1 \mathrm{mv}$ ), como descrito por Tilley (1992).

Cada animal recebeu acepromazina ${ }^{1}, 0,03 \mathrm{mg} / \mathrm{kg}$, por via intravenosa, sendo esse protocolo ansiolítico previamente testado (Cavalcanti et al., 2005; Melo et al., 2006). Dez minutos após,

${ }^{1}$ Univet SA - São Paulo, Brasil. foram realizadas novas medidas de pressão arterial como a descrita anteriormente, contadas as freqüências cardíaca e respiratória. Os cães foram posicionados em decúbito lateral, por contenção manual, para a realização do exame ecodopplercardiográfico, utilizando aparelho Toshiba SSH-140.

Os animais que apresentaram alterações nos exames foram excluídos do experimento. Para a realização do modo bidimensional, seguiram-se as recomendações do Colégio Americano de Medicina Interna Veterinária e do Colégio de Cardiologia Veterinária (Thomas et al., 1993). Foram avaliadas as câmaras cardíacas e a contratilidade miocárdica.

No modo $M$, realizaram-se as medidas e os cálculos da porcentagem de encurtamento sistólico do diâmetro ventricular esquerdo $(\% \Delta \mathrm{d})$ e da fração de ejeção (FE), segundo Boon et al. (1983).

As velocidades da via de saída do ventrículo direito (Vsvd) foram feitas em corte paraesternal direito na região de base cardíaca. As velocidades da via de saída do ventrículo esquerdo (Vsve) e o tempo de ejeção (TE) foram efetuados em corte apical esquerdo - cinco câmaras (Bonagura et al., 1998). 


\section{Cavalcanti et al.}

Conforme descrito por Bonagura et al. (1998), em corte apical esquerdo - quatro câmaras, foram estudados os fluxos das valvas mitral e tricúspide. O tempo de relaxamento isovolumétrico (TRIV) foi obtido no corte apical esquerdo - cinco câmaras. Analisaram-se o tempo de desaceleração da onda E da valva mitral (TDE), os picos de velocidade das ondas de enchimento inicial das valvas mitral e da tricúspide (Em e Et, respectivamente) e os picos de velocidade de enchimento tardio da valva mitral (Am) e da tricúpide (At). Ainda, nesse corte, foi analisado o fluxo das veias pulmonares com o volume da amostra do doppler pulsado na região superior do átrio esquerdo, próximo à entrada das veias esquerda e direita do lobo caudal pulmonar. Após a obtenção desse fluxo, avaliaram-se os picos de velocidade das ondas sistólica (S), diastólica (D) e de reversão atrial (RA) (Bonagura et al., 1998).

Com o auxílio do doppler em cores associado ao modo $\mathrm{M}$, mediu-se a velocidade de propagação de fluxo mitral $(\mathrm{Vp})$, no corte paraesternal esquerdo apical - quatro câmaras, semelhante ao realizado por Garcia et al. (2000), com transdutor de varredura setorial eletrônica de 5,0 Mhz. Durante o exame ECO, realizava-se acompanhamento eletrocardiográfico no monitor; os eletrodos foram colocados no membro anterior esquerdo e nos membros posteriores, como citado por Tilley (1992). Gravaram-se todos os exames em fitas de vídeocassete para posteriores avaliações.

Foram descritas as médias, os desvios-padrão e os coeficientes de variação dos índices ecodopplercardiográficos. Os dados quantitativos de pressão arterial, a freqüência respiratória, a freqüência cardíaca, antes e após a aplicação do tranqüilizante, e a análise entre examinadores dos índices $\mathrm{Vp}$, TDE, TRIV, \% $\% \mathrm{D}$ e FE foram feitos pelo método de comparação de pares a $5 \%$ do teste t de Student.

Foi estimada a correlação da $\mathrm{Vp}$ com os índices FE, $\% \Delta \mathrm{D}$, TRIV, Em, Am, E/Am, TDE, S, D e RA. As correlações foram avaliadas a $5 \%$.

\section{RESULTADOS E DISCUSSÃO}

Houve diminuição das pressões, da freqüência respiratória (FR) e da freqüência cardíaca (FC) (Tab. 1 e 2). A diminuição significativa da freqüência respiratória deveu-se ao fato de a maioria dos cães $(76 \%)$ apresentar-se taquipnéica ao exame físico. Os cães apresentaram valores pressóricos normais antes da aplicação da acepromazina (Tab. 2). O tranqüilizante permitiu contenção manual leve dos animais e diminuição de artefatos na realização do exame ecodopplercardiográfico, sendo a metodologia de tranqüilização igual à testada por Cavalcanti et al. (2005).

Tabela 1. Freqüências respiratória (FR) e cardíaca (FC), medidas antes e após a aplicação da acepromazina, em 36 cães da raça Boxer

\begin{tabular}{ccc}
\hline & Freq. respiratória & Freq. cardíaca \\
\hline & $\overline{\mathrm{X}} \pm \mathrm{DP}(\mathrm{mpm})$ & $\overline{\mathrm{X}} \pm \mathrm{DP}(\mathrm{mpm})$ \\
\hline $\begin{array}{c}\text { Antes } \\
\text { Após 10min. } \\
\text { minutos após }\end{array}$ & $217,37 \pm 69,85 \mathrm{a}$ & $143,81 \pm 23,56 \mathrm{a}$ \\
& $92,14 \pm 76,94 \mathrm{~b}$ & $98,75 \pm 21,39 \mathrm{~b}$ \\
\hline
\end{tabular}

Valores seguidos por letras distintas na coluna diferem entre si pelo teste $\mathrm{t}(\mathrm{P}<0,05)$.

mpm: movimentos por minuto; bpm: batimentos por minuto.

Tabela 2. Pressões arteriais sistólica, diastólica média antes e após a aplicação da acepromazina em 36 cães da raça Boxer

\begin{tabular}{cccc}
\hline & Sistólica & \multicolumn{1}{c}{ Diastólica } & Média \\
& $\overline{\mathrm{X}} \pm \mathrm{DP}(\mathrm{mmHg})$ & $\overline{\mathrm{X}} \pm \mathrm{DP}(\mathrm{mmHg})$ & $\overline{\mathrm{X}} \pm \mathrm{DP}(\mathrm{mmHg})$ \\
\hline Antes & $130,26 \pm 8,46 \mathrm{a}$ & $88,89 \pm 9,24 \mathrm{a}$ & $105,71 \pm 9,08 \mathrm{a}$ \\
10 minutos após & $120,43 \pm 11,61 \mathrm{~b}$ & $70,65 \pm 12,96 \mathrm{~b}$ & $89,87 \pm 12,40 \mathrm{~b}$ \\
\hline
\end{tabular}

Valores seguidos por letras distintas na coluna diferem entre si pelo teste $\mathrm{t}(\mathrm{P}<0,05)$.

Os índices FE, \% $\% \mathrm{D}$, TRIV, TDE e $\mathrm{Vp}$ não diferiram entre os dois examinadores (Tab. 3), possivelmente, devido ao fato de o treinamento do ecocardiografista ter sido feito com ecocardiografista experiente. Os índices que geralmente exibem diferença significativa entre examinadores, segundo Palmieri et al. (2003), são os medidos em tempo.
O estudo ecocardiográfico pode variar entre examinadores, interferindo no diagnóstico (Chetboul et al., 2003). Palmieri et al. (2003), em estudo de parâmetros diastólicos, realizado com dois ecocardiografistas recém-treinados por ecocardiografistas experientes, mostraram que os índices E/Am e Vp apresentaram reprodutibilidade alta, e os índices TRIV e TDE, moderada. 
Tabela 3. Índices ecodopplercardiográficos de acordo com os examinadores, obtidos em 11 cães da raça Boxer

\begin{tabular}{ccccc}
\hline \multirow{2}{*}{ Índice } & \multicolumn{2}{c}{ Examinador 1 } & \multicolumn{2}{c}{ Examinador 2 } \\
\cline { 2 - 5 } & $\overline{\mathrm{X}} \pm \mathrm{DP}$ & $\mathrm{CV}(\%)$ & $\overline{\mathrm{X}} \pm \mathrm{DP}$ & $\mathrm{CV}(\%)$ \\
\hline FE $(\%)$ & $64,83 \pm 3,58$ & 5,52 & $64,94 \pm 4,17$ & 6,42 \\
$\% \Delta \mathrm{D}$ & $35,27 \pm 2,65$ & 7,51 & $35,30 \pm 3,13$ & 8,87 \\
TRIV $(\mathrm{ms})$ & $89,03 \pm 18,06$ & 20,28 & $79,48 \pm 16,61$ & 20,90 \\
TDE $(\mathrm{ms})$ & $85,94 \pm 10,31$ & 15,74 & $87,45 \pm 14,85$ & 16,98 \\
Vp $(\mathrm{cm} / \mathrm{s})$ & $106,45 \pm 12,08$ & 11,35 & $110,01 \pm 7,55$ & 6,86 \\
\hline
\end{tabular}

Os valores na linha não diferem entre si ( $\mathrm{P}>0,05 \%)$

FE: fração de ejeção; \% $\% \mathrm{D}$ : percentual de encurtamento sistólico do ventrículo esquerdo; TRIV: tempo de relaxamento isovolumétrico; TDE: tempo de desaceleração da onda E mitral; Vp: velocidade de propagação do influxo mitral.

Considera-se a avaliação diastólica dos parâmetros ecodopplercardiográficos reproduzível.

Foram obtidas as médias, o desvio-padrão e o coeficiente de variação de todas as medidas ecodopplercardiográficas (Tab. 4).

Tabela 4. Médias, desvio-padrão (DP) e coeficientes de variação (CV) dos índices ecodopplercardiográficos avaliados em 36 cães da raça Boxer

\begin{tabular}{lcc}
\hline Índice & $\overline{\mathrm{X}} \pm \mathrm{DP}$ & $\mathrm{CV}(\%)$ \\
\hline FE $(\%)$ & $65,51 \pm 3,9$ & 5,95 \\
$\% \Delta \mathrm{D}$ & $35,81 \pm 3,09$ & 8,63 \\
Vvsvd (cm/s) & $113,76 \pm 17,98$ & 15,80 \\
Vvsve $(\mathrm{cm} / \mathrm{s})$ & $122,41 \pm 20,66$ & 18,88 \\
TE $(\mathrm{ms})$ & $174,92 \pm 19,82$ & 11,33 \\
TRIV $(\mathrm{ms})$ & $93,46 \pm 19,98$ & 21,38 \\
Em $(\mathrm{cm} / \mathrm{s})$ & $81,47 \pm 18,64$ & 22,88 \\
Am $(\mathrm{cm} / \mathrm{s})$ & $51,71 \pm 11,13$ & 21,52 \\
E/Am & $1,58 \pm 0,19$ & 12,02 \\
TDE $(\mathrm{ms})$ & $83,04 \pm 13,07$ & 15,74 \\
Et $(\mathrm{cm} / \mathrm{s})$ & $71,76 \pm 13,38$ & 18,64 \\
At $(\mathrm{cm} / \mathrm{s})$ & $45,87 \pm 12,35$ & 26,92 \\
E/At & $1,62 \pm 0,29$ & 17,90 \\
S $(\mathrm{cm} / \mathrm{s})$ & $31,41 \pm 6,87$ & 21,87 \\
D $(\mathrm{cm} / \mathrm{s})$ & $73,85 \pm 17,04$ & 23,07 \\
RA $(\mathrm{cm} / \mathrm{s})$ & $28,90 \pm 8,33$ & 28,82 \\
Vp $(\mathrm{cm} / \mathrm{s})$ & $99,73 \pm 16,06$ & 16,10 \\
\hline FE: fras &
\end{tabular}

sistólico do ventrículo esquerdo; Vvsvd: pico da velocidade do fluxo na via de saída do ventrículo direito; Vvsve: pico da velocidade do fluxo na via de saída do ventrículo esquerdo; TE: tempo de ejeção; TRIV: tempo de relaxamento isovolumétrico; Em: pico da velocidade da onda $\mathrm{E}$ mitral; Am: pico da velocidade da onda A mitral; E/Am: relação das ondas E e A mitrais; TDE: tempo de desaceleração da onda E mitral; Et: pico da velocidade da onda E tricúspide; At: pico da velocidade da onda A tricúspide; E/At: relação das ondas E e A tricúspide; S: onda sistólica de enchimento do átrio esquerdo; D: onda diastólica de enchimento atrial esquerdo; RA: onda de reversão atrial; $\mathrm{Vp}$ : velocidade de propagação do influxo mitral.
O TRIV aumenta na doença cardíaca e pode sofrer pseudonormalização. A isquemia por obstrução coronária altera a dinâmica de enchimento ventricular por assincronia no local isquêmico (Mey et al., 2001). Segundo Nunes et al. (2004), pacientes humanos com cardiomiopatia chagásica que morreram em até dois anos apresentaram TRIV de $86,1 \pm 16,9 \mathrm{~ms}$, diferente do TRIV dos pacientes sobreviventes (106,5 $\pm 25,1 \mathrm{~ms})$. O grupo com menor sobrevida apresentou relação E/Am de 2,7 $\pm 0,8$, demonstrando padrão restritivo mitral com TRIV pseudonormalizado.

$\mathrm{Na}$ avaliação do influxo ventricular esquerdo do atual experimento, observou-se que as ondas Em, Am e a relação E/Am (Tab. 4) estão dentro dos valores normais citados na literatura (Abduch, 2004). Em estudo com 24 cães da raça Boxer, Silva (2005) verificou relação E/Am de 1,6 60 ,3, com apenas pequena diferença no desvio-padrão do índice do presente trabalho.

O TDE foi um pouco menor (Tab. 4), mas dentro do desvio-padrão encontrado por Silva (2005), nessa mesma raça. Schober et al. (2003) observaram correlação negativa entre TDE e FC $(\mathrm{r}=-0,54)$.

Há poucos estudos das ondas de enchimento ventricular direito em animais. Neste experimento, observou-se que as ondas Et, At e a relação E/At são semelhantes às citadas na literatura especializada (Abduch, 2004).

Em humanos, as ondas de enchimento ventricular direito variam 30\% com a respiração, e as ondas Em e Am, 10\%. Os índices Em, Am, Et e At não variam com a FC. Em seres 


\section{Cavalcanti et al.}

humanos, as ondas Et e At variam mais e são menos importantes na maioria das doenças cardíacas que as ondas Em e Am (Nagueh et al., 1996).

A onda $\mathrm{S}$ foi menor que a $\mathrm{D}$, mostrando normalidade diastólica ao fluxo das veias pulmonares. Santilli e Bussadori (1998), em estudo de felinos com idade média de 7,75 $\pm 4,51$ anos, obtiveram valores de $39 \pm 12 \mathrm{~cm} / \mathrm{s}$ para a onda $\mathrm{S}, 44 \pm 9 \mathrm{~cm} / \mathrm{s}$ para a $\mathrm{D}$ e $22 \pm 7 \mathrm{~cm} / \mathrm{s}$ para a RA. Esse estudo foi feito em felinos com ampla faixa etária (um a 18 anos) e alta média de idade, o que pode ter levado à diminuição da velocidade da onda D e aumento da onda S. Os picos das ondas de enchimento atrial esquerdo identificam o prejuízo de relaxamento dos seres humanos idosos, por mostrar onda $\mathrm{S}$ maior que a $\mathrm{D}$ (Barbier et al., 2002). Há probabilidade de os cães sadios exibirem relação $\mathrm{S} / \mathrm{D}$ menor que os seres humanos, porém, devido à carência de informações na literatura consultada, mais estudos são necessários.

Mediu-se a Vp (Fig. 2) de acordo com Garcia et al. (2000), que é o modo mais utilizado nos recentes estudos da medicina humana (Palecek et al., 2004). A Vp média observada neste experimento, de $99,73 \pm 16,06 \mathrm{~cm} / \mathrm{s}$, não diferiu entre os sexos e diminuiu com o aumento da idade $(\mathrm{P}<0,01)$ (Tab. 4). A Vp é um dos parâmetros mais importantes deste trabalho e não há, na literatura consultada, padronização da Vp em cães.

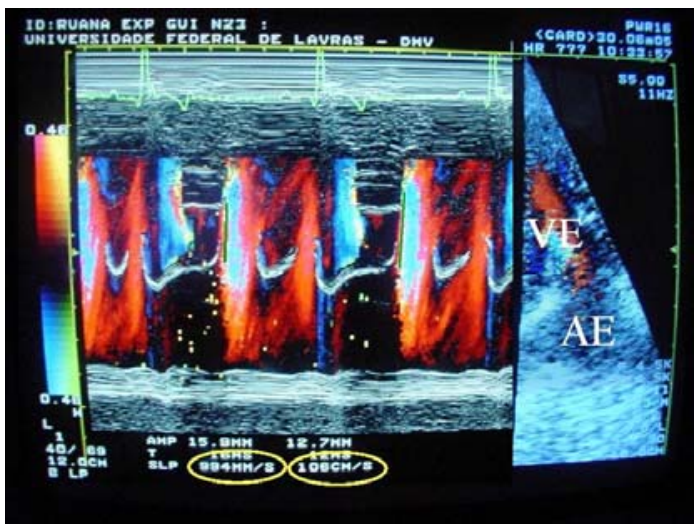

Figura 2. Realização da medida da velocidade de propagação mitral $(\mathrm{Vp})$ em cão da raça boxer. Notar as rampas medidas (ilustradas em verde) com as respectivas velocidades de $99,4 \mathrm{~cm} / \mathrm{s}$ e de $106 \mathrm{~cm} / \mathrm{s}$ (centro das elipses amarelas). AE: átrio esquerdo e VE: ventrículo esquerdo.
Os dois examinadores deste estudo detectaram diminuição da Vp com o aumento da idade, o que também ocorre com seres humanos (Mey et al., 2001). As expressões que representam a diminuição da $\mathrm{Vp}$ foram as retas: $\mathrm{Vp}=110,70-$ $3,47 \mathrm{x}$ idade (em anos), para o examinador 1 , e $\mathrm{Vp}=110-0,20 \mathrm{x}$ idade (em anos), para $\mathrm{o}$ examinador 2. Para a reta do examinador 1 , utilizaram-se 36 cães, e para a do examinador 2 , 11 exames.

Schober et al. (2003) estudaram os índices cardíacos invasivos e por ECO em sete gatos anestesiados, submetidos a alterações hemodinâmicas pelos fármacos esmolol, dobutamina e cilobradina e por infusão de solução de ringer-lactato, até aumento da pressão diastólica final de 5 a $10 \mathrm{mmHg}$. A medida que mais se correlacionou com a tau foi o TRIV $(\mathrm{r}=0,78)$, seguida da $\mathrm{Vp}(\mathrm{r}=-0,68)$. A $\mathrm{Vp}$ não variou com as mudanças na FC nem da pré-carga (Brun et al., 1992).

Barbier et al. (2002) relataram que as diferenças na geometria ventricular, nas dimensões do orifício mitral da cavidade atrial e da cavidade ventricular esquerda, e as diferenças na função sistólica parecem influenciar a $\mathrm{Vp}$. $\mathrm{O}$ atual estudo mostrou independência da $\mathrm{Vp}$ com os principais índices sistólicos ao ECO (Tab. 5), devendo-se avaliar rotineiramente as funções sistólica e diastólica. A avaliação da função diastólica deve ser feita com base nos exames disponíveis associados às avaliações clínicas (Mey et al., 2001). A velocidade de propagação mitral é importante na avaliação diastólica em seres humanos, e deve ser utilizada na medicina veterinária.

Pedone et al. (2004) demonstraram que o aumento da idade em seres humanos prejudica a função diastólica e causa diminuição dos índices Em, E/Am, E ao DT e D, e aumento de Am, da onda A ao DT, RA e S. Estudos realizados com vários grupos etários, em indivíduos humanos saudáveis, confirmaram esses achados e mostraram que o prejuízo não é uniforme com o passar do tempo (Wilkenshoff et al., 2001). Conseqüentemente, os valores de referência de $\mathrm{Vp}$ devem ser registrados para as diferentes faixas etárias, devido à desuniformidade com o avanço da idade. 
Tabela 5. Correlações entre $\mathrm{Vp}$ e os índices ecodopplercardiográficos em 36 cães da raça Boxer

\begin{tabular}{lc}
\hline Índice & Correlação \\
\hline FE $(\%)$ & NS \\
$\% \Delta D$ & NS \\
TRIV $(\mathrm{ms})$ & NS \\
Em $(\mathrm{cm} / \mathrm{s})$ & 0,39 \\
Am $(\mathrm{cm} / \mathrm{s})$ & 0,39 \\
E/Am & NS \\
TDE $(\mathrm{ms})$ & $\mathrm{NS}$ \\
$\mathrm{S}(\mathrm{cm} / \mathrm{s})$ & $\mathrm{NS}$ \\
$\mathrm{D}(\mathrm{cm} / \mathrm{s})$ & 0,39 \\
RA $(\mathrm{cm} / \mathrm{s})$ & $\mathrm{NS}$ \\
\hline
\end{tabular}

FE: fração de ejeção; \% \%D: percentual de encurtamento sistólico do ventrículo esquerdo; TRIV: tempo de relaxamento isovolumétrico; Em: pico da velocidade da onda E mitral; Am: pico da velocidade da onda A mitral; E/Am: relação das ondas E e A mitrais; TDE: tempo de desaceleração da onda $\mathrm{E}$ mitral; S: onda sistólica de enchimento do átrio esquerdo; D: onda diastólica de enchimento atrial esquerdo; RA: onda de reversão atrial. NS: não significativo $(\mathrm{P}>0,05)$.

As correlações entre $\mathrm{Vp}$ e Em, Am e D foram baixas $(\mathrm{r}=0,39)$ e iguais (Tab. 5), e não se observou correlação significativa entre a Vp e os demais índices ecocardiográficos estudados. Essa independência relativa é devido ao fato de a $\mathrm{Vp}$ representar a velocidade com que o vórtice se desloca ao ápice cardíaco, não avaliada por nenhum outro índice.

Conclui-se que a Vp é um índice relativamente independente, reproduzível e importante na avaliação diastólica.

\section{REFERÊNCIAS BIBLIOGRÁFICAS}

ABDUCH, M.C.D. Ecocardiografia. In: CARVALHO, C.F. (Ed).Ultra-sonografia em pequenos animais. São Paulo: Roca, 2004. p.287345.

BARBIER, P.; GRIMALDI, A.; ALIMENTO, M. et al. Echocardiographic determinants of mitral early flor propagation velocity. Am. J. Cardiol., v.90, p.613-619, 2002.

BONAGURA, J.D.; MILLER, M.W.; DARKE, P.G.G. Doppler echocardiography I: pulsed-wave and continuous-wave examinations. Vet. Clin. N. Am.: Small Anim. Pract., v.28, p.1325-1359, 1998.

BOON, J.; WINGFIELD, W.E.; MILLER, C.W. Echocardiographic indices in the normal dog. Vet. Radiol., v.24, p.214-221, 1983.
BRUN, P.; TRIBOUILLOY, C.; DUVAL, A.M. et al. Left ventricular flow propagation during early filling is related to wall relaxation: a color M-mode Doppler analysis. J. Am. Coll. Cardiol., v.20, p.420-432, 1992.

CAVALCANTI, G.A.O.; MUZZI, R.A.L.; MUZZI, L.A.L. et al. Efeito da acepromazina nos parâmetros ecodopplercardiográficos de cães sadios. In: CONGRESSO BRASILEIRO DE VETERINÁRIA, 32., 2005, Uberlândia. Anais... Uberlândia: CONBRAVET, 2005. CD.(Resumo).

CHETBOUL, V.; CONCORDET, D.; POUCHELON, J. L. et al. Effects of Inter- and intra-observer variability on echocardiographic measurements in awake cats. J. Vet. Med. Series A, v.50, p.326-331, 2003.

CRIPPA, L.; FERRO, E.; MELLONI, E. et al. Echocardiographic parameters and indices in the normal Beagle dog. Lab. Anim., v.26, p.190-195, 1992.

GARCIA, M.J.; SMEDIRA, N.G.; GREENBERG, N.L. et al. Color M-mode flow propagation velocity is a preload insensitive index of left ventricular relaxation: animal and human validation. J. Am. Coll. Cardiol., v.35, p.201-208, 2000.

GONÇALVES, A.C.; ORTON, C.; BOON, J.A. et al. Linear, logarithmic, and polynomial models of M-mode echocardiographic measurements in dogs. Am. J. Vet. Res., v.63, p.994-999, 2002.

MELO, M.B.; VEADO, J.C.C.; SILVA, E.F. et al. Dopplerfluxometria das artérias renais: valores normais das velocidades sistólica e diastólica e do índice resistivo nas artérias renais principais. Arq. Bras. Med. Vet. Zootec., v.58, p.691-693, 2006.

MEY, S.D.; SUTTER, J.D.; VIERENDEELS, J. et al. Diastolic filling and pressure imaging: taking advantage of the information in a colour M-mode Doppler image. Eur. J. Echocardiogr., v.2, p.219233, 2001.

NAGUEH, S.F.; KOPELEN, H.A.; ZOGHBI, W.A. et al. Relation of mean right atrial pressure to echocardiographic and Doppler parameters of right atrial and right ventricular function. Circulation, v.93, p.1160-1169, 1996.

NUNES, M.C.P.; BARBOSA, M.M.; ROCHA, M.O.C. Valor prognóstico da disfunção diastólica em pacientes com miocardiopatia dilatada chagásica. Rev. Bras. Ecocardiogr., v.17, p.15-22, 2004.

PALECEK, T.; LINHART, A.; BULTAS, J. et al. Comparison of early diastolic mitral annular 


\section{Cavalcanti et al.}

velocity in detection of mild to moderate left ventricular diastolic function. Eur. J. Echocardiogr., v.5, p.196-204, 2004.

PALMIERI, V.; AREZZI, E.; SABATELLA, M. et al. Interstudy reproducibility of parameters of left ventricular diastolic function: a Doppler echocardiography study. J. Am. Soc. Echocardiogr., v.16, p.1128-1135, 2003.

PEDONE, M.D.; CASTRO, I.; HATEM, D. et al. Variações de parâmetros da função diastólica do ventriculo esquerdo de acordo com a idade através da ecocardiografia com Doppler tecidual. Arq. Brasil. Cardiol., v.83, p.462-465, 2004.

SANTILLI, R.A.; BUSSADORI, C. Doppler Echocardiographic study of left ventricular diastole in non-anesthetized healthy cats. Vet. J., v.156, p.203-215, 1998.

SCHOBER, K.E.; FUENTES, V.L.; BONAGURA, J.D. Comparison between invasive hemodynamic measurements and noninvasive assessment of left ventricular diastolic function by use of Doppler echocardiography in healthy anesthetized cats. Am. J. Vet. Res., v.64, p.93-103, 2003.

SILVA, E. F. Índices ecodopplercardiográficos de função ventricular esquerda em cães das raças Boxer e Schnauzer miniatura. $2005.33 \mathrm{f}$. Dissertação (Mestrado) - Escola de Veterinária,
Universidade Federal de Minas Gerais, Belo Horizonte.

TAKATSUJI, H.; MIKAMI, T.; URASAWA, K. et al. A new approach for evaluation of left ventricular diastolic function: spatial and temporal analysis of left ventricular filling flow propagation by Color M-mode Doppler echocardiography. J. Am. Coll. Cardiol., v.27, p.365-371, 1996.

TIDHOLM, A.; SVENSSON, H.; SYLVÉN, C. Survival and prognostic factors in 189 dogs with dilated cardiomyopathy. J. Am. Hosp. Assoc., v.33, p.364-368, 1997.

TILLEY, L. P. (Ed). Essentials of canine and feline eletrocardiography. 3.ed. Philadelphia: Lea \& Febiger, 1992. 470p.

THOMAS, W.P.; GABER, C.E.; JACOBS, G.J. et al. Recommendations for standards in transthoracic two-dimensional echocardiography in the dog and cat. The echocardiography Committee of the Specialty of Cardiology, American College of Veterinary Internal Medicine. J. Vet. Int. Med., v.7, p.247-252, 1993.

WILKENSHOFF, U. M.; HATLE, L.; SOVANY, A. et al. Age-dependent changes in regional diastolic function evaluated by color Doppler myocardial imaging: a comparison with pulsed Doppler indexes of global function. J. Am. Soc. Echocardiogr., v.14, p.959-969, 2001. 